\title{
VOLUME ESTIMATION FROM SINGLE IMAGES: AN APPLICATION TO PANCREATIC ISLETS
}

\author{
JIŘÍ DVOŘÁK ${ }^{\otimes, 1,2}$, JAN ŠVIHLÍK ${ }^{2,3,4}$, JAN KYBIC², BARBORA RADOCHOVÁ ${ }^{5}$, JIŘÍ \\ JANÁČEK ${ }^{5}$, JAROMÍR KUKAL ${ }^{6}$, JIŘí BOROVEC ${ }^{2}$ AND DAVID HABART ${ }^{7}$
}

${ }^{1}$ Department of Probability and Mathematical Statistics, Faculty of Mathematics and Physics, Charles University in Prague, Sokolovská 83, Prague, Czech Republic; ${ }^{2}$ Biomedical Imaging Algorithms (BIA) group, Center for Machine Perception, Faculty of Electrical Engineering, Czech Technical University in Prague, Technická 2, Prague, Czech Republic; ${ }^{3}$ Department of Computing and Control Engineering, Faculty of Chemical Engineering, University of Chemistry and Technology, Technická 5, Prague, Czech Republic; ${ }^{4}$ Multimedia Technology Group, Department of Radioelectronics, Faculty of Electrical Engineering, Czech Technical University in Prague, Technická 2, Prague, Czech Republic; ${ }^{5}$ Department of Biomathematics, Institute of Physiology of the Czech Academy of Sciences, Vídeňská 1083, Prague, Czech Republic; ${ }^{6}$ Department of Software Engineering, Faculty of Nuclear Sciences and Physical Engineering, Czech Technical University in Prague, Trojanova 13, Prague, Czech Republic; ${ }^{7}$ Institute for Clinical and Experimental Medicine, Vídeňská 1958/9, Prague, Czech Republic

e-mail: dvorak@karlin.mff.cuni.cz, jan.svihlik@fel.cvut.cz, kybic@fel.cvut.cz, barbora.radochova@fgu.cas.cz, jiri.janacek@fgu.cas.cz, jaromir.kukal@fjfi.cvut.cz,jiri.borovec@ fel.cvut.cz, david.habart@ikem.cz (Received December 12, 2017; revised June 1, 2018; accepted June 27, 2018)

\begin{abstract}
The present paper deals with the problem of volume estimation of individual objects from a single $2 \mathrm{D}$ view. Our main application is volume estimation of pancreatic (Langerhans) islets and the single 2D view constraint comes from the time and equipment limitations of the standard clinical procedure.

Two main approaches are followed in this paper. First, two regression-based methods are proposed, using a set of simple shape descriptors of the segmented image of the islet. Second, two example-based methods are proposed, based on a database of islets with known volume. For training and evaluation, islet volumes were determined by OPT microscopy and a stereological volume estimation using the so-called Fakir probes.

The performance of the single image volume estimation methods is studied on a set of 99 islets from human donors. Further experiments were also performed on a stone dataset and on synthetic 3D shapes, generated using a flexible stochastic particle model. The proposed methods are fast and the experimental results show that in most situations the proposed methods perform significantly better than the methods currently used in clinical practice, which are based on simple spherical or ellipsoidal models.
\end{abstract}

Keywords: Fakir probes, pancreatic islets, single image, volume estimation, 2D projection.

\section{INTRODUCTION}

Pancreatic islets can be isolated from a surgically removed pancreas, to be used to treat diabetes by either allogeneic or autologous islet transplantation, respectively (Shapiro et al., 2000; Suszynski et al., 2014), which is a less invasive treatment than a full pancreas transplantation. Clinical outcome of such treatment is known to depend on several variables, including the total islet volume in the graft relative to the body weight of the recipient and the islet size distribution (Lehmann et al., 2007; Suszynski et al., 2014). It is therefore desirable to characterize these properties before the transplantation, ideally using an automatic method (Buchwald et al., 2016; Habart et al., 2016).

The standard approach is to take a few samples from the graft, stain them for insulin producing cells, acquire 2D images using a standard microscope, identify the islets, and estimate their volumes assuming a spherical or ellipsoidal shape. The accuracy is not very high, as the actual islet shape is more variable. While it is not possible to acquire 3D images of the islets during the surgery because of logistical, financial, and time constraints, it is possible to acquire such 3D images for a limited number of islets for the purpose of method development. We applied optical projection tomography (Alanentalo et al., 2007) to individual islets and used the acquired images to obtain ground truth islet volumes.

From the stereology point of view, the task is to estimate the volume of an object from a known population given its one $2 \mathrm{D}$ image. This is a very challenging and ill-defined task, and only a few methods were proposed in the literature (see below). In particular, we assume that binary segmentations 
of the individual islets are available and that these 2D segmentations correspond to thresholded weighted projections of the 3D objects (see below for details). In this study we use manual segmentations for the estimation and compare the results with those based on automatic segmentations of the islet images (see below).

There are several non-standard aspects of this problem in comparison to standard stereology tasks (Baddeley, Jensen, 2005; Howard, Reed, 2005). First, the population of objects is very heterogeneous, we cannot assume they are multiple observations of essentially the same object. Second, the islet orientation is not isotropically random but some orientations are preferred due to islets floating in the liquid medium or lying on the glass bottom (depending on the relative density). Consequently, some parts of the islets may never be observed. Third, we observe neither a parallel projection, nor a crosssection but a convolution with the microscope point spread function, which is something between the two. It resembles a parallel projection but with increasing blur and decreasing influence of points away from the focal plane. Moreover, as the islets are relatively thick and opaque, the interior of the objects cannot be observed. We therefore assume the objects to be homogeneous and only use information from the shape of the $2 \mathrm{D}$ projection boundary.

The classical Ricordi method (Ricordi et al., 1990) used in clinical practice quantizes manually estimated islet diameters into bins. A volume corresponding to each bin is calculated assuming a spherical shape. More advanced approaches fit an ellipse to the 2D shape and assume the islet to be a prolate ellipsoid with the longest axis parallel to the observation plane (Girman et al., 2003). However, the real shapes of the pancreatic islets are not always well represented by these simple models.

A regression approach similar to ours but with different descriptors was used to estimate limestone particle masses in the construction industry (Banta et al., 2003). Chaarani et al. (2013) report a correlation between volume and area measurements for brain ventricles but do not attempt a regression. Multicellular cancer spheroid volumes are estimated using a method similar to the classical vertical planar rotator method (Piccinini et al., 2016). Other applications of single image volume estimation include food volume estimation, see, e.g., $\mathrm{Xu}$ et al. (2013) or Zhang et al. (2011), using 3D stereo reconstruction or virtual reality model fitting. Stereo reconstruction cannot be used for our application because only one image is available, while islet shapes are not sufficiently distinguished and too variable for the model fitting.

\section{MATERIAL AND METHODS}

\section{PANCREATIC ISLET DATASET}

A sample of human pancreatic islets was obtained from 8 different donors. The total number of islets in this study was 99 . The islets were chosen to cover wide range of sizes and shapes and do not constitute a random sample from an islet population.

The islets were isolated using collagenase based method (Berkova et al., 2005) and immediately placed in a standard cell culture. Fresh individual islets (up to 4 hours after the isolation) were briefly stained with Dithizone (which stains the insuline producing beta cells red) and gently washed in Hank's buffered solution (HBSS) supplemented with $0.2 \%$ albumin. Washed islets were imaged using inverted microscope CXK41 (Olympus), $4 \times$ or $10 \times$ magnification objective, and a $3 \mathrm{MP}$ CMOS camera (Infinity1 or Bresser), in order to obtain a single 2D image (see Fig. 1).

Immediately after the microscopy image acquisition, each individual islet was gently placed into a $2 \%$ agarose with low gelling temperature (A9414, Sigma) at $37^{\circ} \mathrm{C}$ and allowed to solidify at room temperature. The agarose block was mounted on the sample holder of a custom made OPT (optical projection tomography) scanner (Politecnico di Milano) equipped with an EM CCD camera (Andor) and PlanApo Infinity-Corrected objective (10x, Edmund Optics). The scanner is designed to rotate a specimen around the vertical axis and acquire a set of images during one turn. For every islet, 400 image projections were obtained using bright field illumination with a rotation step of $0.9^{\circ}$ and a resolution of $1004 \times 1002$ pixels.

3D volumes were created by a tomographic reconstruction using a free software package NRecon (Skyscan), implementing a convolutionbackprojection reconstruction algorithm (Feldkamp et al., 1984). Islet volume was estimated from the 3D reconstruction to serve as ground truth as described in section "Volume estimation from 3D volumes".

\section{STONE IMAGE DATASET}

We obtained colour images of 100 small stones (one image per stone) lying in a natural position on a flat white surface. The images were taken by a digital camera from a fixed position above the stones. 


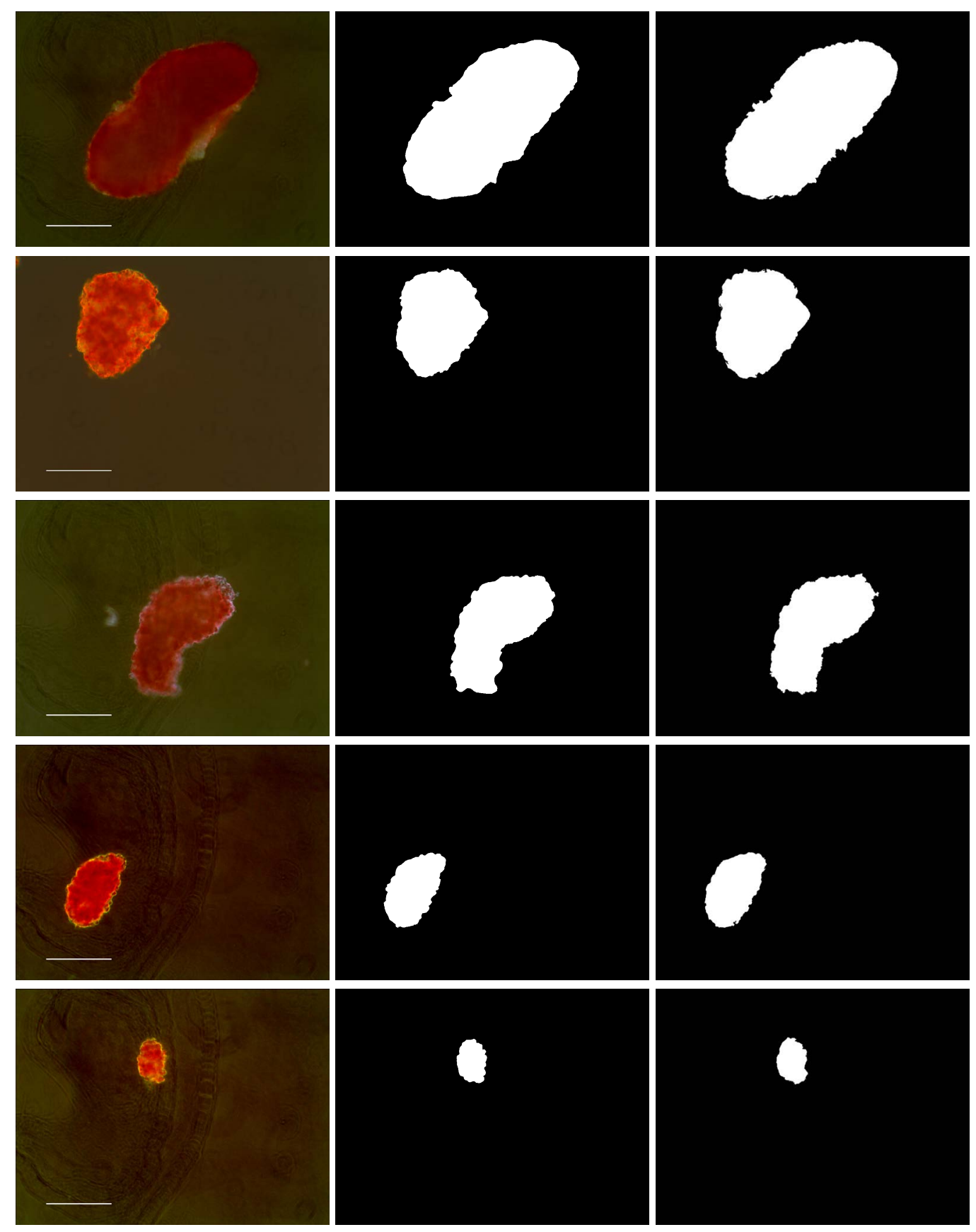

Fig. 1. Examples of the original dark field microscopy images of dithizone-stained individual islets (left), their manual segmentation (middle) and their automatic segmentation (right). The white horizontal bar has length $100 \mu \mathrm{m}$. 


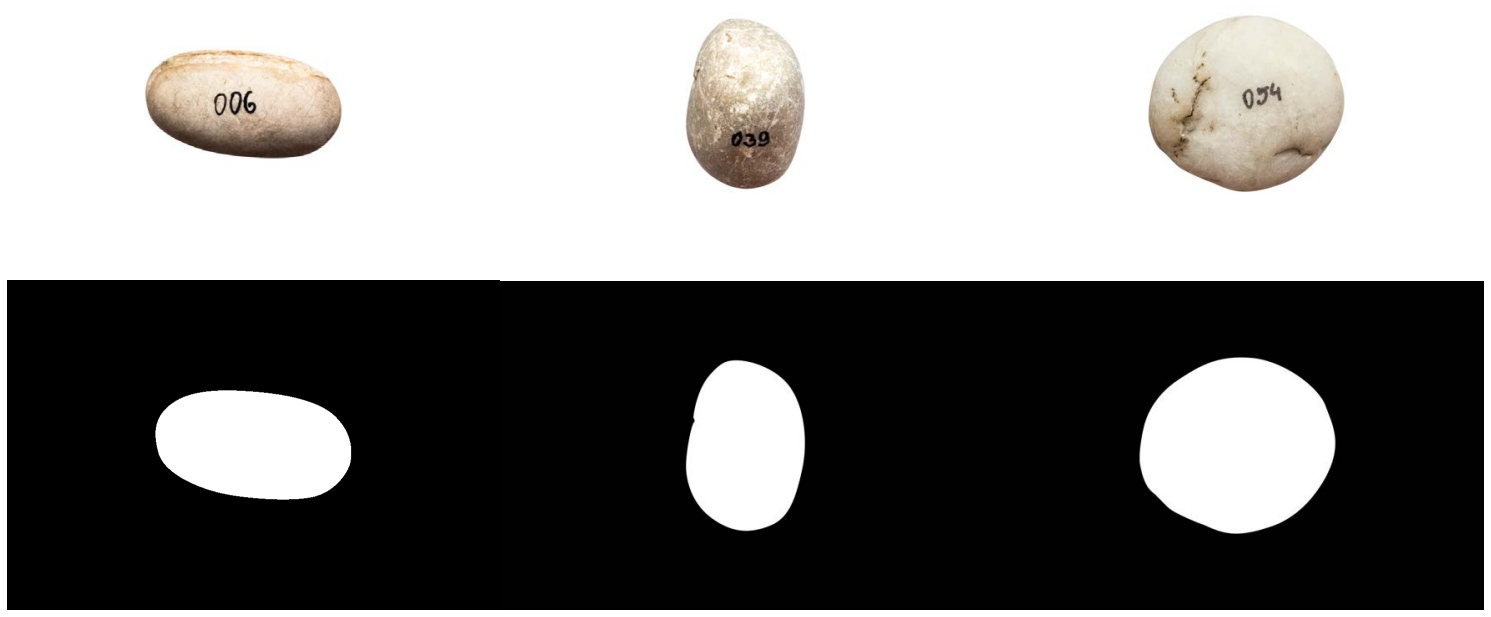

Fig. 2. Examples of stone images (top) and the corresponding segmentations (bottom).

Examples of such image are given in Fig. 2. The true volume of each stone was determined by placing the stone into a graduated cylinder and measuring the volume of water necessary to fill the cylinder to a given level. The volume of a stone used as a ground truth was the mean of three measurements.

\section{SIMULATED DATASETS}

We generated islet-like artificial particles from a flexible stochastic particle model inspired by (Ziegel et al., 2015). The model was based on ellipsoidal particles being inflated by a Lévy random field on the unit sphere. We considered star-shaped particles, i.e., we assumed that all the points on the boundary of the particle can be seen directly from the origin $O$ of the coordinate system. Hence, the boundary of the particle $K$ was fully determined by the so-called radial function $R_{K}(u), u \in \mathbb{S}^{2}$, measuring the distance from $O$ to the boundary of $K$ in the direction $u$. Here $\mathbb{S}^{2}$ denotes the unit sphere in $\mathbb{R}^{3}$.

The model for the radial function of the random particle considered in (Ziegel et al., 2015) is given by $R_{K}(u)=R_{K_{0}}(u) \cdot \varepsilon(u), u \in \mathbb{S}^{2}$, where $R_{K_{0}}$ is the radial function of a fixed star-shaped particle $K_{0}$ and $\varepsilon>0$ is an isotropic Lévy random field on $\mathbb{S}^{2}$, constructed using a Gamma Lévy basis with parameter $\theta>0$ and the von Mises-Fisher kernel with parameter $\kappa>0$ (Ziegel et al., 2015). In our study, we used a slightly modified version of this model, namely $R_{K}(u)=$ $R_{K_{0}}(u) \cdot(1+\varepsilon(u)), u \in \mathbb{S}^{2}$, where $K_{0}$ was an ellipsoid (with semiaxes lengths equal to 1,1 ,and $a$ ) and $\varepsilon$ was a Lévy random field as above. In this model it holds that $K_{0} \subset K$ which seems more appropriate for modeling of pancreatic islets. Note that by fixing two of the three semiaxes lengths, we refrained from evaluating the effect of the absolute size of the particles, making it easier to study the effect of the shape.

In cooperation with two medical experts, we identified four combinations of the model parameters $(a, \kappa, \theta)$ for which the shapes of the simulated particles resemble the shapes of pancreatic islets encountered in practice. The four combinations of parameter values correspond to four sub-populations of real pancreatic islets, described as "small islets" with $\left(a_{1}, \kappa_{1}, \theta_{1}\right)=(6 / 5,5 / 6,5 / 6)$, "medium-sized islets" with $\left(a_{2}, \kappa_{2}, \theta_{2}\right)=(6 / 5,2,2)$, "large and flat islets" with $\left(a_{3}, \kappa_{3}, \theta_{3}\right)=(7 / 10,5 / 2,5)$ and "large and elongated islets" with $\left(a_{4}, \kappa_{4}, \theta_{4}\right)=(18 / 10,5 / 4,2)$. We disregarded other types of real islets which are difficult to describe or simulate, e.g., "large and irregular islets". For each of the four combinations of parameter values we generated 1000 simulated particles, recording their true volumes.

In order to assess the performance of the volume estimation methods also outside the context of smooth particles resembling the shape of pancreatic islets we simulated also 1000 rough particles with sharp points and edges. The particles were generated by forming a convex hull of a random number of points (the number being uniformly distributed between 20 and 50) uniformly distributed in a cube, hence obtaining random polyhedra. They somewhat resemble the limestone particles studied in Banta et al. (2003).

In order to mimic the 2D imaging of real objects (islets, stones), we determined for each simulated particle its orientation in the three-dimensional space with the lowest possible potential energy with respect to the horizontal plane. With this orientation we 
(a)
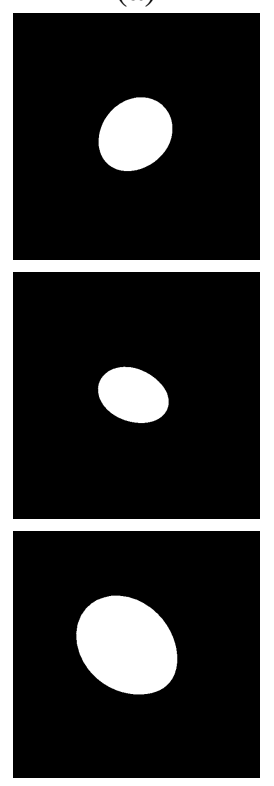

(b)
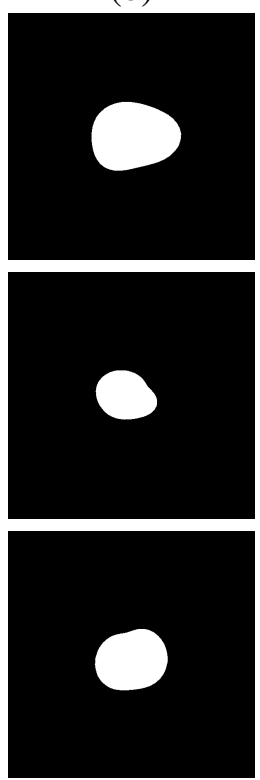

(c)
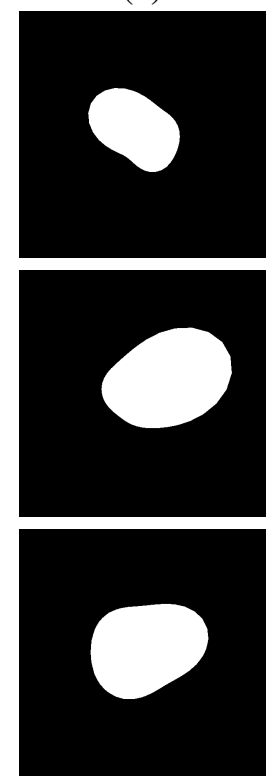

(d)
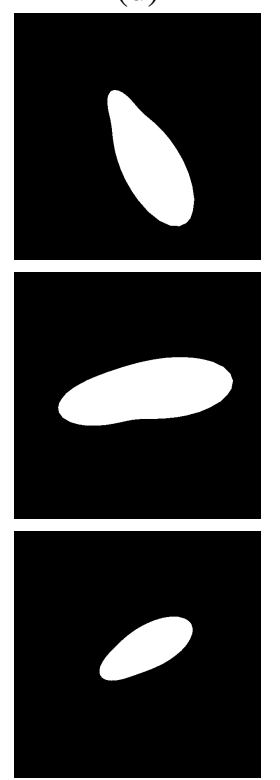

(e)
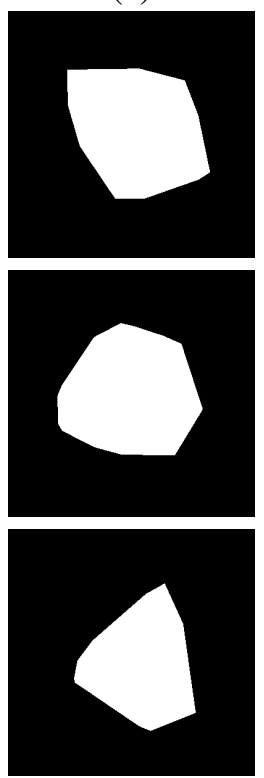

Fig. 3. Examples of projections of the simulated particles. Column (a) "small islets" population, (b) "mediumsized islets" population, (c) "large and flat islets" population, (d) "large and elongated islets" population and (e) "limestone particles" population. Note that the relative sizes of the first four populations of the simulated particles do not reflect the relative sizes of the corresponding populations of human islets. Hence the particles in different columns above look similar in size.

recorded a binary image of the projection of the particle to the horizontal plane. Examples of such images for the four populations of smooth particles and one population of rough particles are given in Fig. 3.

\section{VOLUME ESTIMATION FROM 3D VOLUMES}

To provide a ground truth estimate of the pancreatic islet volumes, we used the Fakir method based on a randomized virtual spatial grid of lines (Kubínová, Janáček, 2001) to find islet volumes from their 3D OPT reconstructions. Islet volumes estimated by this method will be denoted $V_{F}$.

The Fakir method is based on the fact that the mean length of the grid lines inside the objects multiplied by grid length density is equal to the volume of the object. The mean is assessed with respect to the random position of the grid. The method is available as an interactive program.

We also tried to estimate the volume directly from the segmented $3 \mathrm{D}$ reconstruction using level set segmentation as implemented in ImageJ (Yoo, 2004). However, the islets were very large and dense and not enough light was transmitted through, violating the reconstruction assumptions and leading to many reconstruction artifacts, making the segmentation very challenging. Alternatively, the volume can be estimated directly from the projections (Švihlík et al., 2017). This method performed better but was again not sufficiently reliable to serve as the ground truth for our purposes.

\section{AUTOMATIC VOLUME ESTIMATION FROM 2D IMAGES}

All subsequently described methods assume that binary masks of individual objects have been extracted from the images. The segmentation of stone images was performed by thresholding the gray-scale image while the binary masks are available directly for the simulated particles.

Pancreatic islets can be segmented automatically (Habart et al., 2016; Švihlík et al., 2016). In this work we used expert manual segmentations and an automatic supervised segmentation method (Borovec et al., 2017) based on superpixels, color features, random forest classifier, and GraphCut regularization. This enabled evaluation of the effect of segmentation errors. The automatic method was trained on expert manual segmentations using a cross-validation procedure (described in the Results section). Note that because the reference OPT method cannot distinguish between islets and exocrine tissue (red and yellow in 
Fig.1), both were considered as foreground for the purpose of this work.

For easier reference, Table 1 summarizes the names of the volume estimation methods considered in this paper together with their respective abbreviations.

Table 1. Names and abbreviations of volume estimation methods. $V_{F}$ is used here as the ground truth. $V_{S}, V_{E}$ were proposed earlier for pancreatic islets. $V_{P}, V_{B}$ were introduced in other contexts. The remaining methods are proposed here.

\begin{tabular}{lc}
\hline Method & Abbreviation \\
\hline Fakir method & $V_{F}$ \\
Spherical model & $V_{S}$ \\
Ellipsoidal model & $V_{E}$ \\
Piccinini method & $V_{P}$ \\
Banta method & $V_{B}$ \\
Regression method for logarithm of volume & $V_{L S}$ \\
Regression method for volume & $V_{N L S}$ \\
Database method with scaling & $V_{D 1}$ \\
Database method without scaling & $V_{D 2}$ \\
\hline
\end{tabular}

\section{Spherical model}

The most simple approach to volume estimation from a single image is based on the assumption of a spherical shape of the islets (Niclauss et al., 2008). This is a slight and natural modification of the original Ricordi approach (Ricordi et al., 1990). First, the area $A$ of the segmented object is automatically determined and the diameter $d$ of a circle with the same area is calculated as $d=\sqrt{4 A / \pi}$. Then, the volume of the islet is estimated as the volume of a sphere with diameter $d$. Islet volume estimated by this method will be denoted $V_{S}$ :

$$
V_{S}=\frac{\pi}{6} d^{3}=\frac{4}{3 \sqrt{\pi}} A^{3 / 2}
$$

\section{Ellipsoidal model}

This approach generalizes the shape model to a prolate ellipsoid and assumes that its maximal projection is observed. Given the segmented object we first calculate the major and minor axes lengths $a$ and $b, a<b$, of an equivalent fitted ellipse - an ellipse with the same moments up to the second order which is then uniformly scaled to have the same area as the segmented object (as implemented in ImageJ, see also Cramér (1946, p. 283)).

The volume of the islet is then estimated as the volume of a prolate ellipsoid with axes lengths $(a, a, b)$, see Girman et al. (2003). Islet volume estimated by this method will be denoted $V_{E}$ :

$$
V_{E}=\frac{\pi}{6} a^{2} b .
$$

\section{Regression methods}

After fitting the ellipse to the 2D shape as described previously and obtaining axes lengths $a$ and $b, a<b$, the $3 \mathrm{D}$ volume is sought in the form

$$
V=\gamma a^{\alpha} b^{\beta} .
$$

This formula generalizes the spherical and ellipsoidal models described above (thanks to the relation $A=$ $\pi a b / 4)$ and can describe also other situations, such as ellipsoids with the longest axis perpendicular to the imaging plane. We shall describe two different methods of determining the parameters $\alpha, \beta, \gamma$ (other approaches were tested but did not bring any further improvement).

Given a training dataset of $M$ objects described by 3D volumes $V_{i}$ and axis lengths $a_{i}, b_{i}$, the simplest way to estimate the parameters $\alpha, \beta, \gamma$ is to take the logarithm of (3) and minimize the sum of squares:

$$
J_{\mathrm{LS}}=\sum_{i=1}^{M}\left(\log \gamma+\alpha \log a_{i}+\beta \log b_{i}-\log V_{i}\right)^{2} .
$$

This is a standard linear least squares problem in variables $\alpha, \beta$ and $\log \gamma$.

While the previous method is simple and fast, the criterion $J_{\mathrm{LS}}$ being minimized does not necessarily correspond to any practically relevant quantity. As an alternative, we have minimized the sum of squares of the relative volume estimation error:

$$
J_{\mathrm{R}}=\sum_{i=1}^{N}\left(\frac{V_{i}-\gamma a_{i}^{\alpha} b_{i}^{\beta}}{V_{i}}\right)^{2} .
$$

This is a non-linear least squares problem which needs to be solved iteratively, for example by the LevenbergMarquardt method.

Derivatives can be calculated analytically but even the finite difference approximation seems to work well. In practice the optimization procedure converges quickly and reliably and sometimes brings a small accuracy improvement over the linear regression (4). Object volumes calculated by plugging in the estimated values of $\alpha, \beta, \gamma$ minimizing (4) and (5) into (3) will be denoted $V_{L S}$ and $V_{N L S}$, respectively. 


\section{Database method}

In the database method, the estimate of the unknown volume of a new object is calculated from the known volume of the most similar object in the database, with a correction based on the object area in the 2D image. The database contains not only the original images but also their reflections along the horizontal axis, vertical axis, and both axes.

Let the binary database images be denoted $\mathbf{f}_{i}$, $i=1, \ldots, M$, with known volumes $V_{i}$. To estimate a volume of an object from a new image $\mathbf{f}$, we find a database image $\mathbf{f}_{i}$ with the lowest dissimilarity, corresponding to the relative number of pixelwise differences between the two binary images, $i=$ $\arg \min _{j=1, \ldots, M}\left\|\mathbf{f}-\mathbf{f}_{j}\right\| /\|\mathbf{f}\|$, where $\|\mathbf{f}\|$ corresponds to the area of the object in image $\mathbf{f}$.

The volume of the new islet is then estimated as

$$
V_{D}=V_{i}\left(\frac{\|\mathbf{f}\|}{\left\|\mathbf{f}_{\mathbf{i}}\right\|}\right)^{3 / 2}
$$

The scaling factor in (6) relates the volume to cross-section area. The exponent $3 / 2$ is exact for the spherical case, see (1). It corresponds to the situation when the (unobserved) height of the object grows at the same speed as the other two (observed) dimensions.

We consider two versions of the database method. In the first version, both the database and the input images are normalized by rotation and scaling, so that the major axis of the best-fitting ellipse is aligned horizontally and the area of the segmented object is constant. The scaling factor in (6) needs to be calculated from the images before scaling. The estimates obtained by this method will be denoted $V_{D 1}$. In the second version, the images are only rotated but not scaled. The estimates obtained by this method will be denoted $V_{D 2}$.

For illustration of the database method $V_{D 1}$ see the top part of Fig. 4: (a) segmented image of the islet whose volume is to be estimated, (b) the same, rotated and scaled to the reference position, (c) segmented image of the islet identified to be the most similar, (d) the same, rotated and scaled to the reference position. Bottom part of Fig. 4 shows an illustration of $V_{D 2}$ where (e-h) corresponds to (a-d) above with the only difference that scaling is not used.

\section{Other reference methods}

For the sake of comparison we also consider two volume estimation methods based on a single 2D image that were proposed in other contexts and were not used so far for pancreatic islets. The first method (Piccinini et al., 2016) is used in the context of cancer spheroid volume estimation and it is closely related to the classical vertical planar rotator (Jensen, Gundersen, 1993). Details of the method can be found in the original paper (Piccinini et al., 2016). We used the publicly available source code for the Piccinini method and the estimates obtained in this way will be denoted $V_{P}$. Second, we consider the method of Banta et al. (2003). It is a regression-based method with descriptors combining the axes lengths of the best-fitting ellipse and the mean and variance of the distances from the centroid to regularly sampled points on the particle boundary. Details of the method can be found in the original paper (Banta et al., 2003). The estimates obtained by this method will be denoted $V_{B}$.

\section{RESULTS}

Both the regression and database methods need a training dataset of 2D images of objects with known 3D volumes. To evaluate the performance of the estimation methods, we applied a cross-validation scheme, repeatedly dividing the full dataset into a training set and a testing set. For the human islets dataset (99 islets, Fig. 1) we used a 9-fold crossvalidation scheme. For the stones dataset (100 stones, Fig. 2) and the simulated particles (1000 particles in each population, Fig. 3) we applied a 10-fold crossvalidation scheme.

We compared the performance of different volume estimation methods based on the relative bias (RB) and the mean relative squared error (MRSE) defined as follows:

$$
\begin{aligned}
\operatorname{RB}\left(V_{M}\right) & =\frac{1}{N} \sum_{i_{1}}^{N} \frac{V_{M}(i)-V_{F}(i)}{V_{F}(i)}, \\
\operatorname{MRSE}\left(V_{M}\right) & =\sqrt{\frac{1}{N} \sum_{i_{1}}^{N}\left(\frac{V_{M}(i)-V_{F}(i)}{V_{F}(i)}\right)^{2}},
\end{aligned}
$$

where $V_{M}$ is the volume estimation method in question (i.e., $\left.V_{S}, V_{E}, \ldots\right), V_{F}(i)$ is the reference volume of the $i$-th object and $N$ is the number of objects in the dataset. Note the square root in the definition of MRSE; in this way the values are given in the same units as the measurements and the values of RB. Additional robust error measures were calculated but are not reported here since they did not provide any new insight.

We also compared the performance of the volume estimation methods using formal hypotheses testing comparing pairs of different methods. When 

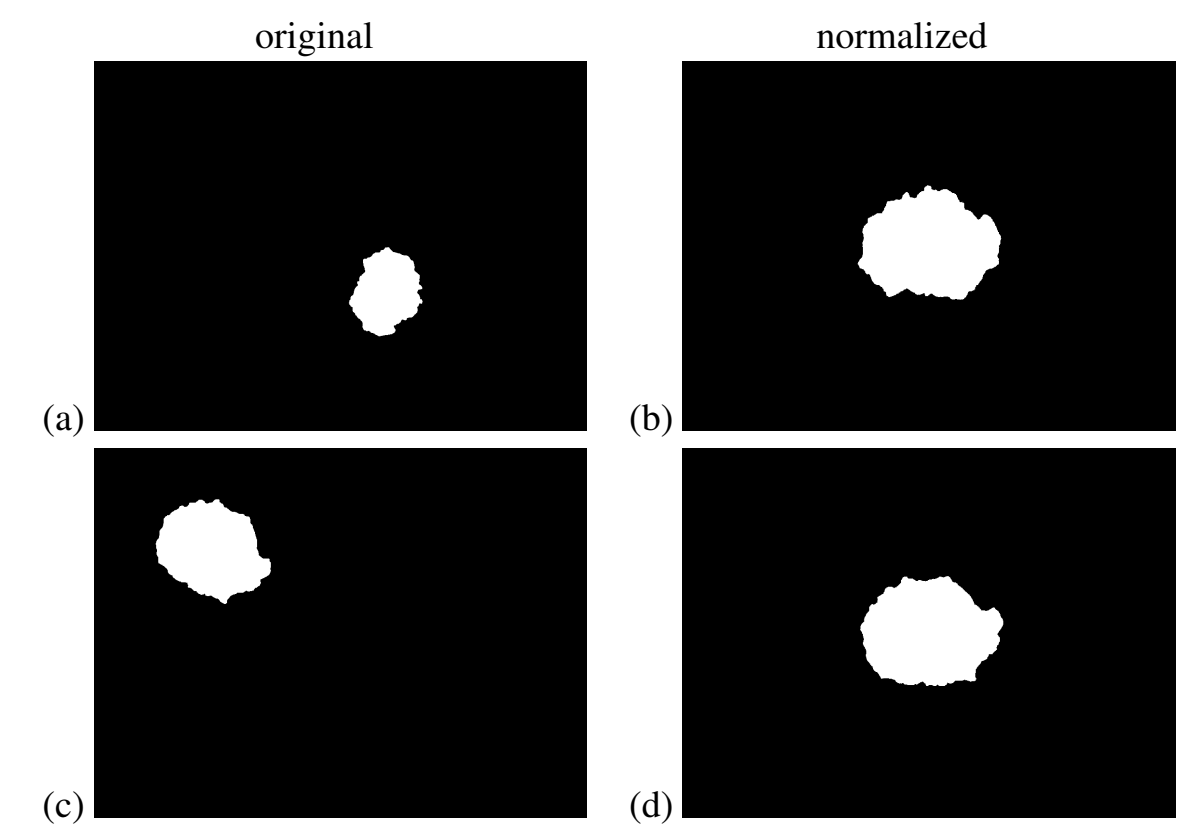

original

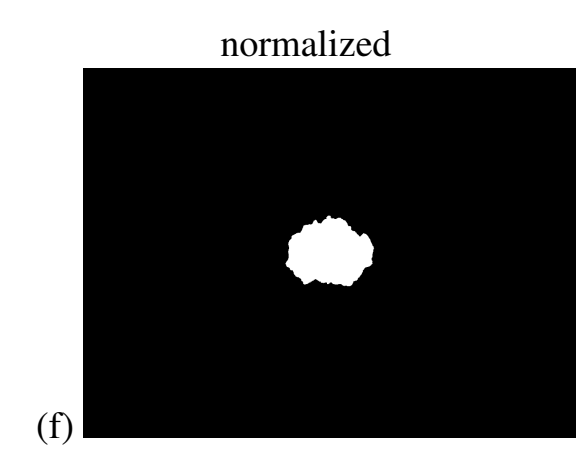

(e)

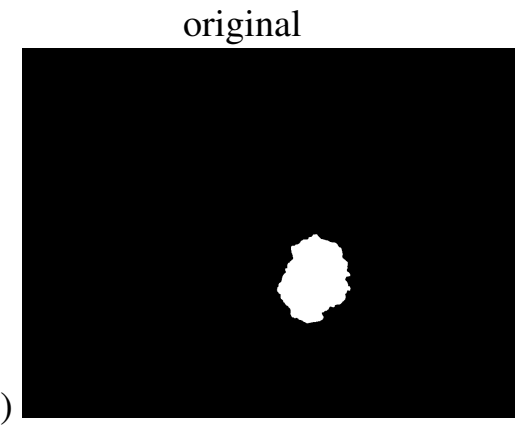

(f)
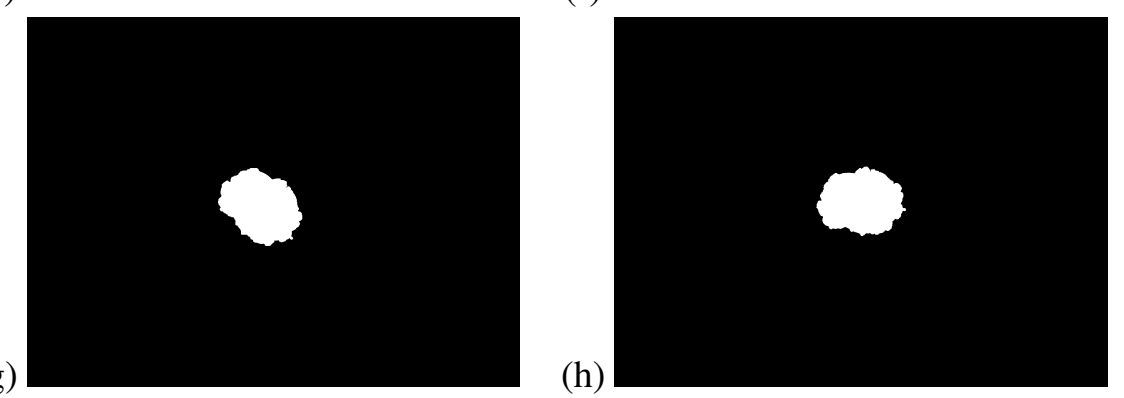

(h)

Fig. 4. Top part $(a-d)$ : illustration of the database method $V_{D 1}$. Bottom part (e-h): illustration of the database method $V_{D 2}$. For details see the main text. 
comparing, e.g., $V_{L S}$ against $V_{S}$ we performed the sign test on the relative error differences

$$
\begin{array}{r}
Z(i)=\frac{\left|V_{L S}(i)-V_{F}(i)\right|}{V_{F}(i)}-\frac{\left|V_{S}(i)-V_{F}(i)\right|}{V_{F}(i)}, \\
\text { for } i=1, \ldots N .
\end{array}
$$

The reason for choosing the sign test over, say, Wilcoxon test was that the individual values of $Z(i)$ are independent but we do not consider them to be identically distributed - the performance of the volume estimation methods may vary across different types of objects (size, shape). Also, the human islets in this study did not constitute a random sample but were chosen in a somewhat systematic way, see section "Pancreatic islet dataset".

The assumptions of classical methods such as ANOVA were not fulfilled either. We used the Holm correction to adapt the set of the resulting $p$-values for multiple testing (Holm, 1979). For each dataset we performed two groups of tests. The first group consisted of 16 one-sided sign tests, with the Holm correction, of the methods proposed in this paper $\left(V_{L S}, V_{N L S}, V_{D 1}, V_{D 2}\right)$ against the reference methods $\left(V_{S}, V_{E}, V_{P}, V_{B}\right)$. One-sided tests were chosen because they enable us, when rejecting the null hypothesis, to establish superiority of our proposed method over a reference method. The second group consisted of 6 two-sided sign tests, with the Holm correction, of all pairs of the methods proposed in this paper. Two-sided tests were chosen because we see no apriori reason why any of the methods should outperform the others. All tests were performed at a 0.05 significance level.

\section{EXPERIMENTS ON THE PANCREATIC ISLET DATASET}

The relative classification error of the automatic segmentation method was $1.5 \%$ with respect to all pixels, which corresponds to $5 \%$ with respect to the number of object (islet) pixels, using the crossvalidation scheme described above.

For the model (3), the regression methods provided the following parameters, estimated from the whole set of 99 islets: $\alpha=1.645, \beta=1.097, \gamma=1.778$ for the least-squares criterion (4) (the $V_{L S}$ method) and $\alpha=1.667, \beta=1.076, \gamma=1.722$ for the relative error criterion (5) (the $V_{N L S}$ method), respectively. It is instructive to see that these values are actually quite far from the classical ellipsoidal model (2) which corresponds to $\alpha=2, \beta=1, \gamma \approx 0.523$. This means that an ellipsoid is a poor approximation of the islet shape.
Tables 2 (manual segmentation) and 3 (automatic segmentation) summarize the numerical characteristics of the different estimators, determined by cross-validation where appropriate. Most of the estimations method perform better on the manual segmentations in terms of MRSE. A notable exception is the Banta method $\left(V_{B}\right)$.

For both manual and automatic segmentation, it is clear that the spherical model $\left(V_{S}\right)$ is too simple to provide useful estimates of islet volumes - it had a large positive bias (approx. 30\%) and, as a result, large MRSE. Compared to the spherical model, the ellipsoidal model $\left(V_{E}\right)$ had a smaller bias $(14-15 \%)$ but the variability was still rather high. The Piccinini method $\left(V_{P}\right)$ performed similarly to $V_{E}$ while the Banta method $\left(V_{B}\right)$ performed slightly better than $V_{E}$.

The regression methods $\left(V_{L S}, V_{N L S}\right)$ were essentially unbiased and their relative error was comparable. Both regression methods outperformed the spherical and ellipsoidal models and the Piccinini and Banta methods in terms of both RB and MRSE. The method with the smallest value of MRSE was $V_{N L S}$.

The database methods did not perform as well as the regression methods. They were slightly better in comparison with the ellipsoidal model in terms of MRSE and they had a smaller bias (4-6\%). They were slightly outperformed by the Banta method.

Fig. 5 shows the estimates for individual islets plotted against their ground truth volume $V_{F}$ for the proposed methods $V_{L S}, V_{N L S}, V_{D 1}, V_{D 2}$ and the reference methods $V_{S}, V_{E}$. This provides graphical comparison of the bias and variability of the estimates. To provide even clearer perspective we report here the number of islets (out of 99) for which it holds that $\mid V_{M}(i)-$ $V_{F}(i) \mid / V_{F}(i) \leq 0.15$, i.e., for which the achieved precision is $15 \%$ or better. Here $V_{M}(i)$ is the volume estimate for the $i$-th islet obtained by a particular method and $V_{F}(i)$ is its ground truth volume. The numbers corresponding to the respective methods are $19\left(V_{S}\right), 46\left(V_{E}\right), 67\left(V_{L S}\right), 71\left(V_{N L S}\right), 58\left(V_{D 1}\right)$ and 51 $\left(V_{D 2}\right)$.

For the manual segmentation, in the first group of sign tests all methods proposed here were found to perform significantly better than $V_{S} ; V_{L S}$ and $V_{N L S}$ were found to perform significantly better than $V_{E}$. In the second group of sign tests a significant difference was found between the pairs $\left(V_{L S}, V_{D 2}\right)$ and $\left(V_{N L S}, V_{D 2}\right)$, the former performing better. No other differences between two methods were significant. The observations made in this paragraph for the manual segmentation apply also to the case of automatic segmentation with a single exception: in the first 

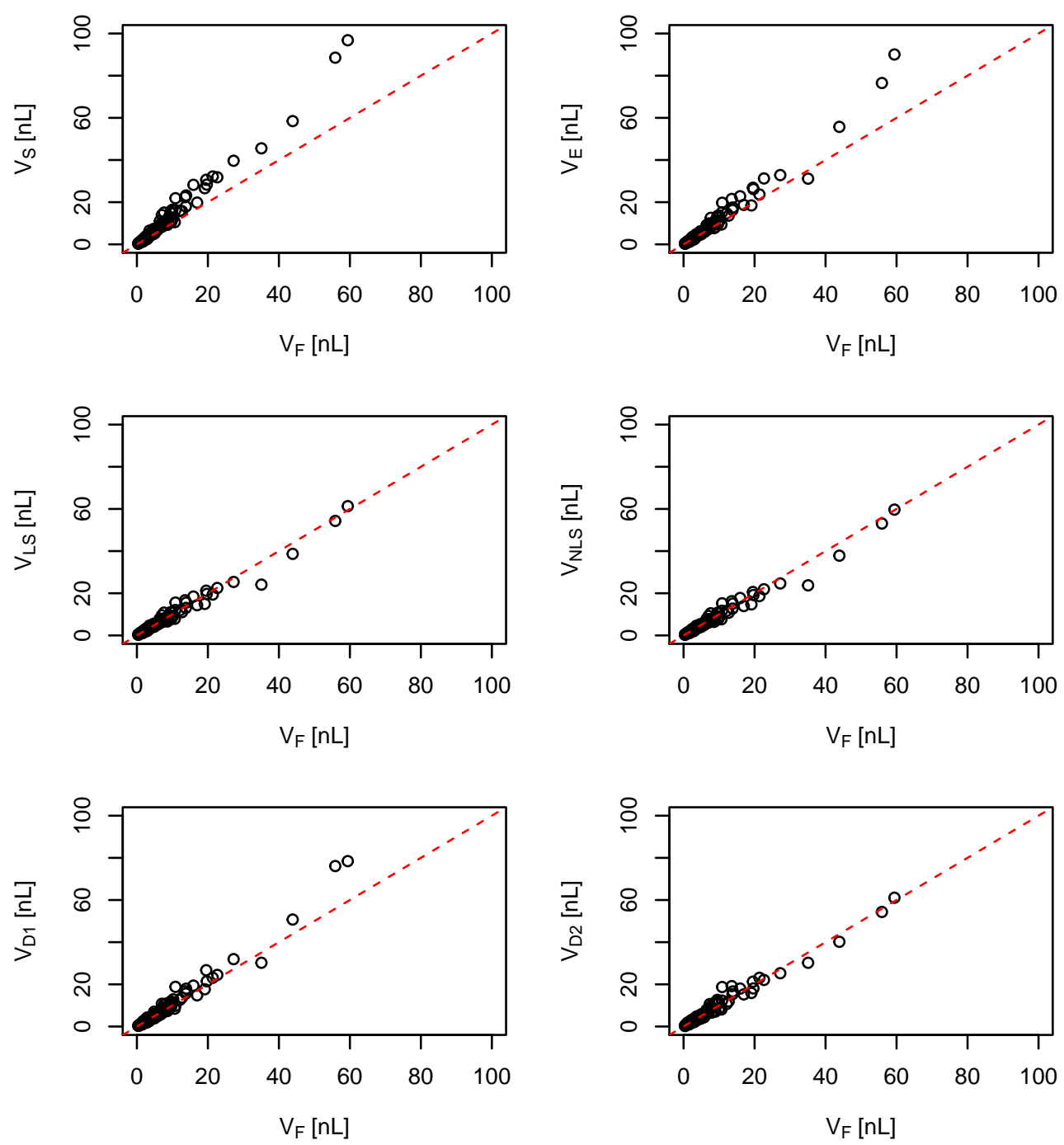

Fig. 5. Volume estimates for individual islets plotted against their ground truth volume $V_{F}$ for the proposed methods $V_{L S}, V_{N L S}, V_{D 1}, V_{D 2}$ and the reference methods $V_{S}, V_{E}$. The dashed line represents the identity mapping $y=x$.

group of sign tests $V_{N L S}$ was not found to perform significantly better than $V_{E}$.

For the two database methods (with manual segmentation) we also investigated the approach of choosing the exponent in (6) based on minimizing the MRSE on training data instead of fixing it at 1.5. For $V_{D 1}$ the optimal scaling factor was 1.43 , resulting in MRSE $=0.188$ instead of 0.206 for 1.5 . For $V_{D 2}$ the optimal scaling factor was 1.25 , resulting in MRSE $=$ 0.217 instead of 0.218 for 1.5 . However, the limited size of our dataset does not allow us to generalize these observations and we therefore kept the exponent at 1.5.

We also investigated the possibility to modify the database method (with manual segmentation) by using $K>1$ nearest neighbors instead of just one and obtaining the volume estimate as a weighted average. The best results were obtained for $K=10$ and exponentially decreasing weights. For the $V_{D 1}$ method we obtained MRSE $=0.192$ instead of 0.206 . For the $V_{D 2}$ method we obtained MRSE $=0.183$ instead of 0.218 . We remark that even with this modification the two database methods did not perform as well as the regression methods in terms of both MRSE and RB.

\section{EXPERIMENTS ON THE STONE DATASET}

Table 4 summarizes the numerical characteristics of the different estimators considered here. While $V_{S}$ and $V_{E}$ exhibited large positive bias and large variability, the regression methods were virtually 
Table 2. Comparison of the volume estimation methods for the islet dataset (manual segmentation).

\begin{tabular}{lcccccccc}
\hline & $V_{S}$ & $V_{E}$ & $V_{L S}$ & \multicolumn{1}{c}{$V_{N L S}$} & $V_{D 1}$ & $V_{D 2}$ & $V_{P}$ & $V_{B}$ \\
\hline RB & 0.304 & 0.140 & 0.012 & -0.017 & 0.052 & 0.055 & 0.114 & 0.030 \\
MRSE & 0.388 & 0.242 & 0.155 & 0.151 & 0.206 & 0.218 & 0.214 & 0.180 \\
\hline
\end{tabular}

Table 3. Comparison of the volume estimation methods for the islet dataset (automatic segmentation).

\begin{tabular}{lcccccccc}
\hline & $V_{S}$ & $V_{E}$ & $V_{L S}$ & \multicolumn{1}{c}{$V_{N L S}$} & $V_{D 1}$ & $V_{D 2}$ & $V_{P}$ & $V_{B}$ \\
\hline RB & 0.313 & 0.152 & 0.012 & -0.019 & 0.061 & 0.036 & 0.117 & 0.029 \\
MRSE & 0.392 & 0.243 & 0.159 & 0.154 & 0.222 & 0.228 & 0.225 & 0.173 \\
\hline
\end{tabular}

Table 4. Comparison of the volume estimation methods for the stones dataset.

\begin{tabular}{lcclrcccc}
\hline & $V_{S}$ & $V_{E}$ & \multicolumn{1}{c}{$V_{L S}$} & \multicolumn{1}{c}{$V_{N L S}$} & $V_{D 1}$ & $V_{D 2}$ & $V_{P}$ & $V_{B}$ \\
\hline RB & 0.858 & 0.630 & 0.0194 & -0.035 & 0.028 & 0.074 & 0.634 & 0.051 \\
MRSE & 0.935 & 0.730 & 0.199 & 0.190 & 0.380 & 0.348 & 0.734 & 0.221 \\
\hline
\end{tabular}

Table 5. Comparison of the volume estimation methods for the simulated datasets. Population $1=$ small islets; 2 = medium-size islets; 3 = large and flat islets; $4=$ large and elongated islets; 5 = rough (limestone) particles .

\begin{tabular}{lcccrcccc}
\hline Population 1 & $V_{S}$ & $V_{E}$ & $V_{L S}$ & $V_{N L S}$ & $V_{D 1}$ & $V_{D 2}$ & $V_{P}$ & $V_{B}$ \\
\hline RB & 0.162 & 0.049 & 0.000 & -0.001 & 0.001 & 0.002 & 0.047 & 0.001 \\
MRSE & 0.165 & 0.057 & 0.023 & 0.023 & 0.032 & 0.032 & 0.055 & 0.024 \\
\hline Population 2 & $V_{S}$ & $V_{E}$ & $V_{L S}$ & $V_{N L S}$ & $V_{D 1}$ & $V_{D 2}$ & $V_{P}$ & $V_{B}$ \\
\hline RB & 0.365 & 0.161 & 0.002 & -0.004 & 0.008 & 0.009 & 0.157 & 0.005 \\
MRSE & 0.380 & 0.186 & 0.067 & 0.067 & 0.091 & 0.092 & 0.182 & 0.071 \\
\hline Population 3 & $V_{S}$ & $V_{E}$ & $V_{L S}$ & $V_{N L S}$ & $V_{D 1}$ & $V_{D 2}$ & $V_{P}$ & $V_{B}$ \\
\hline RB & 0.798 & 0.605 & 0.003 & -0.006 & 0.013 & 0.014 & 0.605 & 0.007 \\
MRSE & 0.813 & 0.622 & 0.078 & 0.077 & 0.113 & 0.114 & 0.622 & 0.085 \\
\hline Population 4 & $V_{S}$ & $V_{E}$ & $V_{L S}$ & $V_{N L S}$ & $V_{D 1}$ & $V_{D 2}$ & $V_{P}$ & $V_{B}$ \\
\hline RB & 0.669 & 0.072 & 0.001 & -0.001 & 0.002 & 0.003 & 0.070 & 0.001 \\
MRSE & 0.677 & 0.085 & 0.036 & 0.036 & 0.046 & 0.049 & 0.083 & 0.077 \\
\hline Population 5 & $V_{S}$ & $V_{E}$ & $V_{L S}$ & $V_{N L S}$ & $V_{D 1}$ & $V_{D 2}$ & $V_{P}$ & $V_{B}$ \\
\hline RB & 0.355 & 0.257 & 0.020 & -0.027 & 0.043 & 0.043 & 0.258 & 0.027 \\
MRSE & 0.428 & 0.335 & 0.214 & 0.164 & 0.227 & 0.217 & 0.335 & 0.176 \\
\hline
\end{tabular}


unbiased and showed rather small MRSE. The database methods again exhibited small positive bias and values of MRSE about twice as high as the regression methods but smaller than $V_{S}$ and $V_{E}$. The Piccinini method $V_{P}$ performed similarly to $V_{E}$; the Banta method $V_{B}$ performed slightly worse than the proposed regression methods.

In the first group of tests all methods proposed here were found to perform significantly better than three of the reference methods $\left(V_{S}, V_{E}, V_{P}\right)$. No significant differences were found between the proposed methods and the $V_{B}$ method. In the second group of tests no significant difference was found between any pair of methods proposed in this paper.

\section{EXPERIMENTS ON SIMULATED DATASETS}

Table 5 summarizes the numerical performance characteristics of the different estimators on the five populations in the simulated datasets.

We first discuss the populations 1 to 4 (smooth, resembling the shapes of real islets). In general, the spherical and ellipsoidal models again exhibited large positive bias and large variability, higher when the population deviated more from the shape assumptions of the model (e.g., in population 3 with large and flat islets the ellipsoidal model overestimated the volume systematically because the unobserved height of the particles was smaller than the observed minor semiaxis length). On the other hand, population 4 with large and elongated particles fitted the assumption of the ellipsoidal model rather well and resulted in only a small positive bias (approx. 7\%) and a rather low variability. Again, the $V_{P}$ method performed very similarly to the $V_{E}$ method in all situations. Another general observation is that the regression methods, the database methods and the $V_{B}$ method were virtually unbiased and that the two proposed regression methods outperformed the two database methods and the $V_{B}$ method in terms of MRSE. The $V_{B}$ method outperformed the database methods in terms of MRSE except for the population 4. The differences in numerical characteristics of $V_{L S}$ and $V_{N L S}$ were negligible for all the four populations. The same applies to $V_{D 1}$ and $V_{D 2}$. It is interesting to note that the most challenging population for the estimation methods was population 3 with flat particles.

In the first group of sign tests all methods proposed here were found to perform significantly better than three of the reference methods $\left(V_{S}, V_{E}, V_{P}\right)$ for all populations of smooth simulated particles. The $V_{L S}$ method was found to perform significantly better than $V_{B}$ in all populations. The $V_{N L S}$ method was found to perform significantly better than $V_{B}$ in populations 3 and 4. The database methods $V_{D 1}, V_{D 2}$ were found to perform significantly better than $V_{B}$ for population 4 .

The second group of sign tests identified no significant difference between $V_{D 1}$ and $V_{D 2}$ (all populations) and between $V_{L S}$ and $V_{N L S}$ (populations 1,3 and 4). In all other cases the difference between the methods was significant. However, bear in mind that the sample size was 10 times larger than for the islets and stones datasets. In cases where the difference was found to be significant, the regression methods always outperformed the database methods and $V_{L S}$ outperformed $V_{N L S}$ in terms of the number of times the $Z(i)$ 's were positive/negative, see (9).

Concerning the rough particles (population 5), we observed again that the spherical and ellipsoidal model (methods $V_{S}, V_{E}$ ) did not capture well the shape of the particles, resulting in large positive bias and large variability of the estimates. The same holds true for the $V_{P}$ method. The database methods $V_{D 1}, V_{D 2}$ were again slightly outperformed by the regression methods $V_{L S}, V_{N L S}$. It is interesting to note that the $V_{B}$ method performed very well in this case. This is not surprising as it was developed for volume estimation of rough limestone particles. Nevertheless, the $V_{B}$ method was outperformed by the $V_{N L S}$ method proposed here.

In the first group of sign tests, for the rough particles, all methods proposed here were found to perform significantly better than three of the reference methods $\left(V_{S}, V_{E}, V_{P}\right)$ and they did not perform significantly better than $V_{B}$ - they in fact performed worse. In the second group of sign tests $V_{N L S}$ performed significantly better than $V_{L S}, V_{D 1}, V_{D 2}$ and $V_{D 2}$ performed significantly better than $V_{D 1}$. No other significant differences were found.

\section{DISCUSSION}

In this study we proposed four methods for volume estimation of individual objects from their single 2D images. All proposed methods $\left(V_{L S}, V_{N L S}, V_{D 1}, V_{D 2}\right)$ outperformed the currently used method for islet volume estimation $\left(V_{E}\right)$ in terms of relative bias and mean relative squared error (MRSE) on all three datasets. On the pancreatic islets dataset, as well as on the stones dataset and the simulated particles dataset, we demonstrated a significant performance improvement over $V_{E}$ for the two proposed regression methods methods $\left(V_{L S}, V_{N L S}\right)$ by means of sign tests with a correction for multiple comparisons. The database methods $\left(V_{D 1}, V_{D 2}\right)$ performed significantly better than $V_{E}$ in the stones and simulated particles datasets, but not on the pancreatic islets. 
From the regression methods we suggest using $V_{N L S}$ as it explains the relevant quantity (volume) directly, as opposed to $V_{L S}$ which explains the logarithm of the volume instead. The experiments on the stone dataset and simulated particles indicate that the $V_{N L S}$ method is able to capture enough information about the population in question to provide reliable estimates. However, if speed and simplicity is preferred, then $V_{L S}$ can also be used with a negligible loss of accuracy. The database methods were outperformed by the regression methods in all situations. We assume this might be because of the high heterogeneity of the objects and the relatively small database size.

\section{ACKNOWLEDGEMENTS}

This work has been supported by the grant 17-15361S of the Czech Science Foundation, by the project LM2015062 of the Ministry of Education, Youth and Sports, by the projects CZ.02.1.01/0.0/0.0/16_013/0001775 (funded by OP RDE) and CZ.02.1.01/0.0/0.0/16_019/0000765 (Research Center for Informatics).

\section{REFERENCES}

Alanentalo T, Asayesh A, Morrison H, Lorén CE, Holmberg D, Sharpe J, Ahlgren U (2007). Tomographic molecular imaging and 3D quantification within adult mouse organs. Nat Methods 4:31-3.

Baddeley A, Jensen EBV (2005). Stereology for statisticians. Boca Raton: Chapman \& Hall/CRC.

Banta L, Cheng K, Zaniewski J (2003). Estimation of limestone particle mass from 2D images. Powder Technol 132:184-9.

Berková Z, Kř́̌ž J, Girman P, Zacharovová K, Koblas T, Dovolilová E, Saudek F (2005). Vitality of pancreatic islets labeled for magnetic resonance imaging with iron particles. Transplant Proc 37:3496-8.

Borovec J, Švihlík J, Kybic J, Habart D (2017). Supervised and unsupervised segmentation using superpixels, model estimation, and graph cut. J Electron Imaging 26:061610.

Buchwald P, Bernal A, Echeverri F, Tamayo-Garcia A, Linetsky E, Ricordi C (2016). Fully automated islet cell counter (ICC) for the assessment of islet mass, purity, and size distribution by digital image analysis. Cell Transplant 25:1747-61.

Chaarani B, Capel C, Zmudka J, Daouk J, Fichten A, Gondry-Jouet C, Bouzerar R, Balédent O (2013). Estimation of the lateral ventricles volumes from a 2D image and its relationship with cerebrospinal fluid flow. BioMed Res Int 2013:215989.

Cramér H (1946). Mathematical methods of statistics. Princeton: Princeton University Press.

Feldkamp LA, Davis LC, Kress JW (1984). Practical cone-beam algorithm. J Opt Soc Am A 1:612-9.

Girman P, Kř́ž J, Friedmanský J, Saudek F (2003). Digital imaging as a possible approach in evaluation of islet yield. Cell Transplant 12:12933.

Habart D, Švihlík J, Schier J, Cahová M, Girman P, Zacharovová K, Berková Z, Kř̌̃ž J, Fabryová E, Kosinová L, Papáčková Z, Kybic J, Saudek F (2016). Automated analysis of microscopic images of isolated pancreatic islets. Cell Transplant 25:2145-56.

Holm S (1979). A simple sequentially rejective multiple test procedure. Scand J Stat 6:65-70.

Howard CV, Reed MG (2005). Unbiased stereology, 2nd Ed. Liverpool: QTP Publications.

Jensen EBV, Gundersen HJG (1993). The rotator. J Microsc 170:35-44.

Kubínová L, Janáček J (2001). Confocal microscopy and stereology: Estimating volume, number, surface area and length by virtual test probes applied to three-dimensional images. Microsc Res Tech 53:425-35.

Lehmann R, Zuellig RA, Kugelmeier P, Baenninger PB, Moritz W, Perren A, Clavien PA, Weber M, Spinas GA (2007). Superiority of small islets in human islet transplantation. Diabetes 56:594-603.

Niclauss N, Sgroi A, Morel P, Baertschiger R, Armanet M, Wojtusciszyn A, Parnaud G, Muller Y, Berney T, Bosco D (2008). Computer-assisted digital image analysis to quantify the mass and purity of isolated human islets before transplantation. Transplantation 86:1603-9.

Piccinini F, Tesei A, Bevilacqua A (2016). Singleimage based methods used for non-invasive volume estimation of cancer spheroids: a practical assessing approach based on entrylevel equipment. Comput Methods Programs Biomed 135:51-60.

Ricordi C, Gray DW, Hering BJ, Kaufman DB, Warnock GL, Kneteman NM, Lake SP, London NJ, Socci C, Alejandro R et al (1990). Islet isolation assessment in man and large animals. Acta Diabetol Lat 27:185-95.

Shapiro AM, Lakey JR, Ryan EA, Korbutt GS, Toth E, Warnock GL, Kneteman NM, Rajotte RV (2000). Islet transplantation in seven patients with 
type 1 diabetes mellitus using a glucocorticoidfree immunosuppressive regimen. $\mathrm{N}$ Engl $\mathrm{J}$ Med 343:230-8.

Suszynski TM, Wilhelm JJ, Radosevich DM, Balamurugan AN, Sutherland DE, Beilman GJ, Dunn TB, Chinnakotla S, Pruett TL, Vickers SM, Hering BJ, Papas KK, Bellin MD (2014). Islet size index as a predictor of outcomes in clinical islet autotransplantation. Transplantation 97:1286-91.

Švihlík J, Kybic J, Habart D (2016). Automated separation of merged Langerhans islets. In: Medical Imaging 2016: Image Processing. Proc SPIE 9784:978438.

Švihlík J, Kybic J, Habart D, Hlushak H, Dvořák J, Radochová B (2017). Langerhans islet volume estimation from 3D optical projection tomography. In: Chen CS, Lu J, Ma KK (eds). Computer Vision
- ACCV 2016. Lect Not Comput Sci 10117:58394.

Xu C, He Y, Parra A, Delp E, Khanna N, Boushey C (2013). Image-based food volume estimation. In: Proc ACM 5th Int Works Multimed Cooking Eating Activ (CEA '13) 75-80.

Yoo TS (2004). Insight into images: Principles and practice for segmentation, registration, and image analysis. New York: CRC Press.

Zhang Z, Yang Y, Yue Y (2011). Food volume estimation from a single image using virtual reality technology. In: Proc 2011 IEEE 37th Ann Northeast Bioeng Conf (2 p.).

Ziegel JF, Nyengaard JR, Jensen EBV (2015). Estimating particle shape and orientation using volume tensors. Scand J Stat 42:813-31. 